$\underline{\text { Preprint typeset in JHEP style - HYPER VERSION }}$

DFTT-05/2008

AEI-2008-014

\title{
The Supermembrane with Central Charges on a G2 Manifold
}

\author{
A. Belhaj ${ }^{1}$, M.P. Garcia del Moral $^{2}$, A. Restuccia ${ }^{3,4}$, A. Segui ${ }^{1}$, J.P. Veiro ${ }^{5},{ }^{*}$ \\ 1 Departamento de Física Teórica, Universidad de Zaragoza \\ 12, Pedro Cerbuna, 50009-Zaragoza, Spain. \\ 2 Dipartimento di Fisica Teorica, Università di Torino \\ \& INFN - Sezione di Torino; Via P. Giuria 1; I-10125 Torino, Italy. \\ ${ }^{3}$ Max-Planck-Institut für Gravitationphysik, Albert-Einstein-Institut \\ Mülenberg 1, D-14476 Potsdam, Germany. \\ ${ }^{4}$ Departamento de Física, Universidad Simón Bolívar \\ Apartado 89000, Caracas 1080-A, Venezuela. \\ 5 Departamento de Matemáticas Puras y Aplicadas, Universidad Simón Bolívar \\ Apartado 89000, Caracas 1080-A, Venezuela.
}

\begin{abstract}
We construct the 11D supermembrane with topological central charges induced through an irreducible winding on a G2 manifold realized from the $T^{7} / Z_{2}^{3}$ orbifold construction. The hamiltonian $H$ of the theory on a $T^{7}$ target has a discrete spectrum. Within the discrete symmetries of $H$ associated to large diffeomorphisms, the $Z_{2} \times Z_{2} \times Z_{2}$ group of automorphisms of the quaternionic subspaces preserving the octonionic structure is relevant. By performing the corresponding identification on the target space, the supermembrane may be formulated on a G2 manifold, preserving the discretness of its supersymmetric spectrum. The corresponding 4D low energy effective field theory has $N=1$ supersymmetry.
\end{abstract}

KEYWORDS: Supermembrane, minimal immersions, G2 manifolds, supersymmetric spectrum.

*E-mail: belhaj@unizar.es; garcia@to.infn.it; restucci@aei.mpg.de, arestu@usb.ve; segui@unizar.es; pierre@ma.usb.ve 


\section{Contents}

1. Introduction 1

2. M2 with central charges associated to an irreducible winding 3

3. G2 compactification in M-theory 7

3.1 G2 manifolds 0

$3.2 Z_{2} \times Z_{2} \times Z_{2}$ symmetries of the G2 structure 8

4. MIM2 on a G2 manifold 10

4.1 Minimal immersions on the target space 10

4.2 Configuration space 14

4.3 Connection with Calabi-Yau compactifications 16

5. Quantum properties of the supersymmetric theory 18

6. Phenomenological analysis of the MIM2 on this G2 manifold 19

77. Discussion and conclusions 21

\section{Introduction}

Compactifications of the low energy limit of M-theory to four dimensions (4D) have received much attention during the past years. Special interest has been given to the compactification over real manifolds of dimension seven, $\mathrm{X} 7$, with non trivial holonomy. This interest is due to the fact that these manifolds provide a potential point of contact with low energy semi-realistic physics from M-theory [1, 2].

Our goal along this paper will be to show the quantization of the supersymmetric action of the supermembrane, restricted by a topological condition, on a particular G2 manifold. This is the only (known) quantum consistent way of doing it starting from the supermembrane, there is no other way so far. We will show that this model represents a starting point of a new kind of supersymmetric quantum consistent models with potentially interesting properties from a phenomenological point of view. 
Let us make a brief review of the main properties of the compactification of Mtheory on G2 manifolds. In particular, one can obtain 4D $N=1$ supersymmetry by compactifying M-theory on X7 with G2 holonomy group [3, 4, 5]. In this regard, the 4D $N=1$ resulting models generically depend on geometric properties of $\mathrm{X} 7$. For instance, if X7 is smooth, the low energy theory contains, in addition to $N=1$ supergravity, only abelian gauge groups and neutral chiral multiplets. However, non abelian gauge symmetries with charged chiral fermions can be obtained by considering limits where X7 develops singularities [7, 8, 9, 10, 6]. For a review see for example [11].

Besides the ordinary compactification on G2 manifolds, Calabi-Yau flux compactifications and twisted toroidal compactifications have been also studied intensively, see for example, [12]- [21]. Indeed their respectively phenomenological predictions with different signatures on the LHC have also been considered, see [22, 23] for G2 compactifications, and [24] for large volume approach in Calabi-Yau compactifications. They have also been considered as particular cases of non-geometric compactifications. Most of these approaches follow a bottom-up pattern by studying the $N=1$ gauged supergravity potentials in $4 \mathrm{D}$ and trying to perform the uplift to M-theory. Other compactifications from 11D supergravity with fluxes have also been done in a top-down approach [20, 21].

Recently new types of compactifications have appeared involving twisted boundary conditions or non-trivial fiber bundles over some compact manifolds (with or without singularities), T- foldings [25]. In this way, the metric and the gauge field forms get generically entangled. This kind of compactifications is called nongeometric [26, 27]. Some of these non-geometric compactifications are related with the ordinary ones by dualities. The nontriviality of the fiber bundle guarantees the existence of a monodromy, but usually due to the lack of 1-cycles inside a Calabi-Yau makes it necessary to include singularities. A simple example of these T-foldings is the twisted tori. It is a Scherk-Schwarz compactification of the $11 \mathrm{D}$ supergravity theory with twisted boundary conditions that allows to have a nontrivial monodromy, see for example in connection with G2 compactifications [28]. When the base space is a torus it is no longer necessary to include singularities in order to have a nontrivial monodromy [26, 27]. These twisted compactifications can have a geometrical dual which corresponds to an orbifold plus a shift, also known as asymmetric orbifold [29].

The compactification with a duality twist is more general than the orbifold compactification because it can be carried without restricting the moduli to special variations. The moduli can have nontrivial variation along the circle in the spacetime. However, the orbifold is possible for special values of the moduli where the lattice admits a symmetry and the class of allowed rotations is finite. All of the lattices 
admit a $Z_{2}$ symmetry as the discrete subgroup of the $S L(2, Z)$ of the torus, and for those cases the geometrical dual exists [30].

The 11D supermembrane is one of the basic elements of M-theory [31, 32]. Classically, it is unstable due to the existence of string-like spikes that leave the energy unchanged. At the quantum level, its supersymmetric spectrum is continuous and the theory was interpreted as a second quantized theory [33, 34]. Compactification on $S^{1}$ has been explored in order to see if the continuity of the spectrum is broken by the winding. It has been argued not to be the case [35] due to the presence of string-like spikes in the spectrum. In [36, 37, 38, 39, 40, 42] the minimally immersed supermembrane compactified on a torus associated to the existence of irreducible winding (MIM2) has been found. It is associated to nontrivial fiber bundles defined on Riemann surfaces. This MIM2 is classically stable since there are no singular configurations with zero energy. The quantum spectrum of the theory is purely discrete with finite multiplicity [38, 39, 40, 43, 41]. The theory of the supermembrane minimally immersed in a 7 torus has recently been found in 445]. It has a $N=1$ supersymmetry in $4 \mathrm{D}$. A natural question is to look for a connection with a compactification of the supermembrane in a nontrivial background of type of G2 manifold. In this paper we will be concerned with a full-flegged sector of M-theory which is the quantum supermembrane theory minimally immersed MIM2 on a $T^{6} \times S^{1}$. This type of compactifications contains nontrivial discrete twists on the fibers as remanent discrete symmetries of the hamiltonian. We will show by identifying those symmetries on the target that the MIM2 can admit a compactification on a G2 manifold.

The paper is structured as follows: In Section 2 we introduce the supermembrane with central charges minimally immersed (M2MI) on a $T^{6} \times S^{1}$. We summarize its main spectral properties and symmetries. In Section 3 we recall the main properties of the compactification on G2 manifolds. In Section 4 we construct the MIM2 on a $\frac{T^{7}}{Z_{2}^{3}}$, by studying the minimal immersions of the MIM2 on that target, finding the configuration space of states: the untwisted and twisted sectors of the theory. We also study its connections with Calabi-Yau compactifications. In Section 5 we present our discussions and final conclusions.

\section{M2 with central charges associated to an irreducible wind- ing}

We start this section by recalling that the hamiltonian of the $D=11$ supermembrane [31] in the light cone gauge (LCG) reads as

$$
\int_{\Sigma} \sqrt{W}\left(\frac{1}{2}\left(\frac{P_{M}}{\sqrt{W}}\right)^{2}+\frac{1}{4}\left\{X^{M}, X^{N}\right\}^{2}+\text { Fermionic terms }\right) .
$$


$M$ runs for $M=1, \ldots, 9$ corresponding to the transverse coordinates of the base manifold $R \times \Sigma$. $\Sigma$ is a Riemann surface of genus $g$. The term $\frac{P_{M}}{\sqrt{W}}$ is the canonical momentum density and $\left\{X^{M}, X^{N}\right\}$ is given by

$$
\left\{X^{M}, X^{N}\right\}=\frac{\epsilon^{a b}}{\sqrt{W(\sigma)}} \partial_{a} X^{M} \partial_{b} X^{N},
$$

where $a, b=1,2$ and $\sigma^{a}$ are local coordinates over $\Sigma$. $W(\sigma)$ is a scalar density introduced in the LCG fixing procedure. The former hamiltonian is subject to the two following constraints

$$
\begin{gathered}
\phi_{1}:=d\left(\frac{P_{M}}{\sqrt{W}} d X^{M}\right)=0 \\
\phi_{2}:=\oint_{C_{s}} \frac{P_{M}}{\sqrt{W}} d X^{M}=0,
\end{gathered}
$$

where $C_{s}, s=1, \ldots, 2 g$ is a basis of 1 -dimensional cycles on $\Sigma$. $\phi_{1}$ and $\phi_{2}$ are generators of area preserving diffeomorphisms. When the target manifold is simply connected, the one-forms $d X^{M}$ are exact.

The $S U(N)$ regularized model obtained from (2.1) was shown to have continuous spectrum from $[0, \infty),[33$, 34, 32]. This property of the theory relies on two basic facts: supersymmetry and the presence of classical singular configurations. The latter is related to string-like spikes which appear with zero cost energy. These spikes do not preserve neither the topology of the world-volume nor the number of particles. These properties do not disappear when the theory is compactified and the spectrum remains continuous [35].

To get a 4 dimensional model, we need a target space as $M_{4} \times T^{6} \times S^{1}$. In this way, the configuration maps satisfy the following condition on $T^{6}$

$$
\oint_{c_{s}} d X^{r}=2 \pi S_{s}^{r} R^{r} \quad r, s=1, \ldots, 6 .
$$

On the circle, we have the constraint

$$
\oint_{c_{s}} d X^{7}=2 \pi L_{s} R_{7}
$$

while for non compact directions, we have

$$
\oint_{c_{s}} d X^{m}=0 \quad m=8,9 .
$$

$S_{s}^{r}, L_{s} \in Z$ and $R_{r}, R_{7}$ represent respectively the radii of the 6-torus and the radius of the circle. We shall now impose a topological irreducible wrapping condition to 
be satisfied by all configurations in the above model. This generates a non-trivial central charge in the $11 \mathrm{D}$ supersymmetric algebra. The topological condition is

$$
I^{r s} \equiv \int_{\Sigma} d X^{r} \wedge d X^{s}=n\left(2 \pi R^{r} R^{s}\right) \omega^{r s}
$$

where $\omega^{r s}$ is a symplectic matrix on the $T^{6}$ which can be taken as

$$
\omega^{r s}=\left(\begin{array}{cccccc}
0 & 1 & & & & \\
-1 & 0 & & & & \\
& & 0 & 1 & & \\
& & -1 & 0 & & \\
& & & & 0 & 1 \\
& & & & -1 & 0
\end{array}\right) .
$$

Each block $M=\left(\begin{array}{cc}0 & 1 \\ -1 & 0\end{array}\right)$ defines a sympletic geometry on a $T^{2}$. It also describes the intersection matrix of the homology basis. If we denote by $a$ and $b$ the two elements of the basis of $T^{2}, M_{a b}$ is defined by the following intersection numbers: $a \cdot b=-b \cdot a=1$ and $a \cdot a=b \cdot b=0$. For simplicity on our analysis we will take $n=1$, the general case only involve some technical additional details.

The above topological condition leads to a $D=11$ supermembrane with nontrivial central charges generated by its wrapping on the compact part of the target space. Since the topological constraint commutes with the rest of the constraints, it represents a sector of the full theory characterized by an integer $n=\operatorname{det} \omega^{r s}$, see [38 for a more general discussion. Under such correspondence there exists a minimal holomorphic immersion from the base to the target manifold. The image of $\Sigma$ under that map is a calibrated submanifold of $T^{6}$. The spectrum of the theory changes dramatically since it has a pure discrete spectrum at the classical and the quantum level [38, 39, 40, 41, 43]; see also 45, 42] ${ }^{1}$.

The model that we study here involves additional symmetries beyond the original ones 41] which will be crucial in our coming discussion. In the following the minimally immersed M2 associated to this sector of the theory will be denoted by MIM2 to distinguish it from the usual one.

We notice that the condition in (2.8) only restricts the values of $S_{s}^{r}$. From equation (2.5) we can see that these values should be integral numbers. The condition in (2.8) can be solved by

$$
d X^{r}=M_{s}^{r} d \widehat{X}^{s}+d A^{r}
$$

\footnotetext{
${ }^{1}$ The geometrical interpretation of this condition has been discussed in previous work 36], 37]
} 
where we have decomposed the closed one-forms $d X^{r}$ into their harmonic plus exact parts. Note that $d \widehat{X}^{s}, s=1, \ldots, 2 g$ is a basis for harmonic one-forms over $\Sigma$. They may be normalized with respect to the associated canonical basis of homology,

$$
\oint_{c_{s}} d \widehat{X}^{r}=\delta_{s}^{r}
$$

We have now considered a Riemann surface with a class of an equivalent canonical basis. The condition in (2.5) leads to

$$
M_{s}^{r}=2 \pi R^{r} S_{s}^{r} .
$$

Imposing the condition in (2.8), we get

$$
S_{t}^{r} \omega^{t u} S_{u}^{s}=\omega^{r s},
$$

which says that $S \in S p(2 g, Z)$. This is the most general map satisfying (2.8).

A sufficient condition in order to have a consistent global construction of the theory, subject to the topological constraint, is to have a surface $\Sigma$ of genus $g$ such that the space of holomorphic one-forms is of the same complex dimension as the flat torus in the target space. This condition ensures the existence of a holomorphic immersion, and so minimal, from $\Sigma$ to $T^{2 g}$ [42]. In [45] we analyzed the theory for genus 3 and the breaking of the SUSY by the ground state (the holomorphic immersion) for genus $1,2,3$. It was also emphasized there that in order to consider the MIM2 from $\Sigma$ to a given target space one should consider all possible immersions, in particular all holomorphic immersions. This consideration will become important in the following sections when we analyse a $\frac{T^{7}}{Z_{2}^{3}}$ target space.

The theory is invariant not only under the diffeomorphisms generated by $\phi_{1}$ and $\phi_{2}$ but also under the diffeomorphisms, which are biholomorphic maps, changing the canonical basis of homology by a modular transformation.

We may always consider a canonical basis such that

$$
d X^{r}=2 \pi R^{r} d \widehat{X^{r}}+d A^{r} .
$$

In this manner, the corresponding degrees of freedom are described exactly by the single-valued fields $A^{r}$. By using the condition in (2.6), we perform a similar decomposition with the remaining 1-form associated to the compactification on $S^{1}$

$$
d X^{7}=2 \pi R L_{s} d \widehat{X}^{s}+d \widehat{\phi}
$$

where $d \widehat{\phi}$ is a new exact 1 -form and $d \widehat{X}^{s}$ are the basis of harmonic forms as before. The final expression of the hamiltonian of the MIM2 wrapped in an irreducible way 
on $T^{6} \times S^{1} 45$ is

$$
\begin{aligned}
H= & \int_{\Sigma} \sqrt{w} d \sigma^{1} \wedge d \sigma^{2}\left[\frac{1}{2}\left(\frac{P_{m}}{\sqrt{W}}\right)^{2}+\frac{1}{2}\left(\frac{\Pi^{r}}{\sqrt{W}}\right)^{2}+\frac{1}{4}\left\{X^{m}, X^{n}\right\}^{2}+\frac{1}{2}\left(\mathcal{D}_{r} X^{m}\right)^{2}\right. \\
& \left.+\frac{1}{4}\left(\mathcal{F}_{r s}\right)^{2}+\frac{1}{2}\left(F_{a b} \frac{\epsilon^{a b}}{\sqrt{W}}\right)^{2}+\frac{1}{8}\left(\frac{\Pi^{c}}{\sqrt{W}} \partial_{c} X^{m}\right)^{2}+\frac{1}{8}\left[\Pi^{c} \partial_{c}\left(\widehat{X}_{r}+A_{r}\right)\right]^{2}\right] \\
& \left.+\Lambda\left(\left\{\frac{P_{m}}{\sqrt{W}}, X^{m}\right\}-\mathcal{D}_{r}\left(\frac{\Pi^{r}}{\sqrt{W}}\right)-\frac{\Pi^{c}}{2 \sqrt{W}} \partial_{c}\left(F_{a b} \frac{\epsilon^{a b}}{\sqrt{W}}\right)\right)+\lambda \partial_{c} \Pi^{c}\right] \\
& +\int_{\Sigma} \sqrt{W}\left[-\bar{\Psi} \Gamma_{-} \Gamma_{r} \mathcal{D}_{r} \Psi+\bar{\Gamma}_{-} \Gamma_{m}\left\{X^{m}, \Psi\right\}+1 / 2 \bar{\Psi} \Gamma_{7} \Pi^{b} \partial_{b} \Psi\right]+\Lambda\left\{\bar{\Psi} \Gamma_{-}, \Psi\right\}
\end{aligned}
$$

where $\mathcal{D}_{r} X^{m}=D_{r} X^{m}+\left\{A_{r}, X^{m}\right\}, \mathcal{F}_{r s}=D_{r} A_{s}-D_{s} A_{r}+\left\{A_{r}, A_{s}\right\}$ and

$D_{r}=2 \pi R^{r} \frac{\epsilon^{a b}}{\sqrt{W}} \partial_{a} \widehat{X}^{r} \partial_{b} . \quad P_{m}$ and $\Pi_{r}$ are the conjugate momenta to $X^{m}$ and $A_{r}$ respectively. $\mathcal{D}_{r}$ and $\mathcal{F}_{r s}$ are the covariant derivative and curvature of a symplectic noncommutative theory [37, 39], constructed from the symplectic structure $\frac{\epsilon^{a b}}{\sqrt{W}}$ introduced by the central charge. The physical degrees of the theory are then described by $X^{m}, A_{r}$, and the corresponding spinorial ones $\Psi_{\alpha}$. They are single valued fields on $\Sigma$.

At this level, one might naturally ask the following question. Does there exist a MIM2 compactified on a seven dimensional manifold with G2 holonomy group? In what follows we address this question using a recent result from algebraic geometry of toroidal compactification in the presence of discrete symmetries.

\section{G2 compactification in M-theory}

As we mentioned in the introduction, a possible way to get four dimensional models with four supercharges is to consider the compactification of M-theory on seven dimensional manifolds with G2 holonomy group ${ }^{2}$ [6, 47, 48, 49]. We will refer to them as G2 manifolds. In this manner, different $N=1$ models in four dimensions depend on the geometric realization of the G2 manifold. As for the Calabi-Yau case, there are many geometric realizations. In what follows we quote some of them [50].

\subsection{G2 manifolds}

Let us consider $R^{7}$ parametrized by $\left(x_{1}, x_{2}, \ldots, x_{7}\right)$. On this space, one can define the metric as $g=d x_{1}^{2}+\ldots .+d x_{7}^{2}$. Reducing the group $S O(7)$ to G2, there is a special real three-form

$$
\Psi=d x_{127}+d x_{135}-d x_{146}-d x_{236}-d x_{245}+d x_{347}+d x_{567}
$$

\footnotetext{
${ }^{2} \mathrm{G} 2$ is a group of dimension 14 and rank 2 .
} 
where $d x_{i j k}$ denotes the exterior form $d x_{i} \wedge d x_{j} \wedge d x_{k}$. This expression for $\Psi$ arises from the fact that G2 is the group of automorphisms for the octonionic algebra structure given by

$$
t_{i} t_{j}=-\delta_{i j}+f_{k i j} t_{k}
$$

which yields the correspondence

$$
f_{k i j} \rightarrow d x_{k i j} .
$$

In general if a seven Riemanian metric admits a covariant constant spinor the holonomy group is G2 and there is exactly one. In such manifolds there exists an orthogonal frame, $\widehat{e}^{i}$, in which the octonionic three form $\phi=f_{i j k} \widehat{e}^{i} \wedge \widehat{e}^{j} \wedge \widehat{e}^{k}$ and its dual are closed. $\phi$ is G2 invariant. It turns out that the simplest example of G2 manifolds, which we are interested in here, is the orbifold realization. Let us consider a 7-tori $T^{7}=R^{7} / Z^{7}$, where now $x$ parameterizes $R / Z$. A G2 manifold can be constructed from an orbifold action $T^{7} / \Gamma$ where $\Gamma$ a discrete subgroup of G2, and hence leaving the above three-form $\Psi$ invariant. A possible choice is given by

$$
\Gamma=Z_{2} \times Z_{2} \times Z_{2}
$$

to be defined in the next section.

\section{$3.2 Z_{2} \times Z_{2} \times Z_{2}$ symmetries of the G2 structure}

The $Z_{2}$ symmetries leaving invariant the 3-form (3.1), which we will consider, change signs on certain elements of the basis for the octonions. A change of sign for one element of the basis condemns the same for other elements. These combinations are given by the multiplication table. For convenience in further identifications we have chosen the multiplication table represented in Figure 1, where the $e_{i}$ are the elements from the basis of the octonions. The result for the multiplication of two elements in the basis is the only other element that shares the line passing through the first two, and the sign is given by the arrows. For example, $e_{6} e_{7}=e_{5}$ while $e_{5} e_{2}=-e_{4}$.

A very quick way to determine such subsets of elements is by considering the canonical quaternionic subspaces of the octonions.

Changing signs for the elements in these subsets or their complements each preserve the octonionic structure. The former maps $\Psi \rightarrow-\Psi$ while the latter leaves $\Psi$ completely unchanged. According to the multiplication table we have chosen, the indexes of the elements from the basis corresponding to these sets are given as follows: These seven transformations obtained by changing signs for the elements on the second file, together with the identity, form a commutative group with eight elements of order 


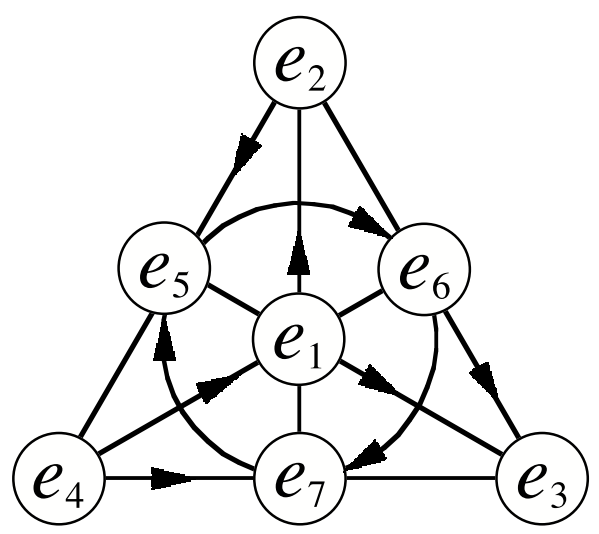

Figure 1: Fano plane representing the multiplication table for the octonions used throughout this paper.

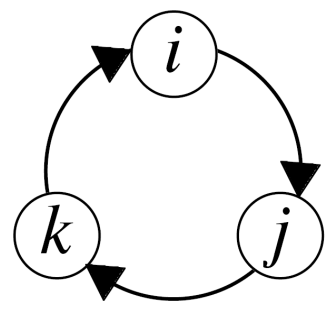

Figure 2: quaternionic diagram.

\begin{tabular}{|l|l|l|l|l|l|l|l|}
\hline$\Psi \rightarrow-\Psi$ & $1,2,7$ & $1,3,5$ & $1,4,6$ & $2,3,6$ & $2,4,5$ & $3,4,7$ & $5,6,7$ \\
\hline$\Psi \rightarrow \Psi$ & $3,4,5,6$ & $2,4,6,7$ & $2,3,5,7$ & $1,4,5,7$ & $1,3,6,7$ & $1,2,5,6$ & $1,2,3,4$ \\
\hline
\end{tabular}

Table 1: Transformations that preserve the octonionic structure.

two. This group is $Z_{2} \times Z_{2} \times Z_{2} \cong Z_{2}^{3}$. There is a nice geometric interpretation for the operation in this group. Given two transformations, they correspond to two quaternionic subspaces of the multiplication table for the octonions and share only one element -see the first row in the previous table. The composition of these transformations is the one related to the only other quaternionic subspace that shares this element in common. Using the same labeling for the multiplication table of the octonions as the one that determines (3.1) we can list all the $Z_{2}$ symmetries that leave invariant the 3 -form $\Psi$ as follows, and, naturally, the identity transformation is in correspondence with $(0,0,0)$.

Aiming towards a $T^{2} \times T^{2} \times T^{2} \times S^{1}$ compact space, we shall identify the coordinates $\left(x_{1}, x_{2}, x_{3}, x_{4}, x_{5}, x_{6}, x_{7}\right)$ with $\left(z_{1}, z_{2}, z_{3}, x_{7}\right) \in \mathbb{C} \times \mathbb{C} \times \mathbb{C} \times \mathbb{R}$ writing $z_{k}=x_{2 k-1}+i x_{2 k}$ for $k=1,2,3$. The transformations given in the previous table are then expressed as in Table 3. All these symmetries can be obtained as composition of the three canonical generators, $(1,0,0),(0,1,0)$, and $(0,0,1)$, for $Z_{2}^{3}$. Nevertheless, there are 28 different subsets of generators for $Z_{2}^{3}$ but all geometrically equivalent. 


\begin{tabular}{|ccc|}
\hline elements that change sign & & the element in $Z_{2}^{3}$ \\
\hline$x_{3}, x_{4}, x_{5}, x_{6}$ & $\longleftrightarrow$ & $(0,1,1)$ \\
$x_{2}, x_{4}, x_{6}, x_{7}$ & $\longleftrightarrow$ & $(1,1,1)$ \\
$x_{2}, x_{3}, x_{5}, x_{7}$ & $\longleftrightarrow$ & $(1,0,0)$ \\
$x_{1}, x_{4}, x_{5}, x_{7}$ & $\longleftrightarrow$ & $(0,1,0)$ \\
$x_{1}, x_{3}, x_{6}, x_{7}$ & $\longleftrightarrow$ & $(0,0,1)$ \\
$x_{1}, x_{2}, x_{5}, x_{6}$ & $\longleftrightarrow$ & $(1,0,1)$ \\
$x_{1}, x_{2}, x_{3}, x_{4}$ & $\longleftrightarrow$ & $(1,1,0)$ \\
\hline
\end{tabular}

Table 2: $Z_{2}^{3}$ transformations preserving G2 structure.

\begin{tabular}{|l|c|}
\hline \multicolumn{1}{|c|}{ symmetry transformation } & the element in $Z_{2}^{3}$ \\
\hline$\left(z_{1}, z_{2}, z_{3}, x_{7}\right) \rightarrow\left(z_{1},-z_{2},-z_{3}, x_{7}\right)$ & $(0,1,1)$ \\
$\left(z_{1}, z_{2}, z_{3}, x_{7}\right) \rightarrow\left(\overline{z_{1}}, \overline{z_{2}}, \overline{z_{3}},-x_{7}\right)$ & $(1,1,1)$ \\
$\left(z_{1}, z_{2}, z_{3}, x_{7}\right) \rightarrow\left(\overline{z_{1}},-\overline{z_{2}},-\overline{z_{3}},-x_{7}\right)$ & $(1,0,0)$ \\
$\left(z_{1}, z_{2}, z_{3}, x_{7}\right) \rightarrow\left(-\overline{z_{1}}, \overline{z_{2}},-\overline{z_{3}},-x_{7}\right)$ & $(0,1,0)$ \\
$\left(z_{1}, z_{2}, z_{3}, x_{7}\right) \rightarrow\left(-\overline{z_{1}},-\overline{z_{2}}, \overline{z_{3}},-x_{7}\right)$ & $(0,0,1)$ \\
$\left(z_{1}, z_{2}, z_{3}, x_{7}\right) \rightarrow\left(-z_{1}, z_{2},-z_{3}, x_{7}\right)$ & $(1,0,1)$ \\
$\left(z_{1}, z_{2}, z_{3}, x_{7}\right) \rightarrow\left(-z_{1},-z_{2}, z_{3}, x_{7}\right)$ & $(1,1,0)$ \\
\hline
\end{tabular}

\section{Table 3:}

\section{MIM2 on a G2 manifold}

In this section we will consider the construction of a MIM2 on a $G_{2}$ manifold. We start from the MIM2 on a seven torus $T^{7}$ and we will perform the identification of the $Z_{2} \times Z_{2} \times Z_{2}$ group, described in Section 3, on the target space.

The MIM2 theory on $T^{7}$ is invariant under the area preserving diffeomorphisms. The ones homotopic to the identity are generated by the area preserving constraints (2.3) and (2.4). The theory is also invariant under large area preserving diffeomorphisms, non-homotopic to the identity, associated to $\operatorname{Sp}(6, Z)$ acting on a Teichmüler space of the moduli space of $g=3$ Riemann surfaces as explained in Section 2. We will now show that the $Z_{2} \times Z_{2} \times Z_{2}$ automorphisms of quaternionic subspaces of the octonionic algebra described in Section 3.2 are also symmetries of the hamiltonian of the MIM2 on $T^{7}$. Moreover, those are the maximal identifications we can perform on the target space preserving $N=1$ susy. We will see that the remaining symmetries of the fiber become spureous whenever the orbifold action on the states is considered.

\subsection{Minimal immersions on the target space}

The maps (2.14,2.15) from the base $\Sigma(\mathrm{g}=3)$ to the compact sector of the target 
space $T^{7}$ decompose into a harmonic part plus an exact one. The harmonic part is a minimal immersion from $\Sigma$ to the compact sector $T^{7}$ of the target space. The requirement introduced in [45] was to consider all possible immersions from the base manifold to the target space. It has a natural interpretation in terms of the existence of fluxes on the compact sector of the target space. In fact, the existence of fluxes is equivalent to the existence of a bundle gerbe or higher order bundle on the target space [54], [55] [56], and [57, [58], [59, [60]. Given a closed p-form $W_{p}$ satisfying the quantization condition

$$
\int_{\Sigma_{p}} W_{p}=2 \pi n
$$

for any $\Sigma_{p}$ submanifold there always exists a bundle gerbe or higher order bundle with its corresponding transition functions on $p-1, \ldots, 1$ forms such that $W_{p}$ is the field strength of a generalized connection. The consistency condition on the transition functions is now satisfied on the overlapping of $p+1$ open sets of an atlas. For the case $p=2$, it is a $U(1)$ principle bundle and the quantization condition ensures the existence of a connection on it such that $W_{2}$ is its curvature. The condition (4.1) must be satisfied for all $\Sigma_{p}$ submanifolds, the integer $n$ may change with $\Sigma_{p}$. If we interpret the central charge condition as a flux condition on the target, we must then impose it for all admissible minimal immersion form $\Sigma$ to $T^{7}$. In the case of the MIM2 on a $T^{7}$ target we should then consider all possible immersions and impose for each of them the topological or central charge condition. This is a geometrical argument emphasizing that we should consider the summation of all possible immersions from $\Sigma$ to the target, see also 61.

We now proceed to consider all possible immersions from $\Sigma$, a genus 3 Riemann surface to $T^{7}=S^{1} \times \cdots \times S^{1}$. The reason to consider a genus 3 surface was explained in Section 2, they are the relevant ones when considering the wrapping of a supermembrane on a $T^{6}$ target. We consider all decompositions of $T^{7}$ into $T^{6} \times S^{1}$, by changing the $S^{1}$ we obtain the complete set of seven sectors. The supermembrane wraps in an irreducible way onto the $T^{6}$, we ensure it by imposing the topological condition on all configurations of the supermembrane on that sector. We distinguish each sector by an integer $i=1, \ldots, 7$ and denote the corresponding maps to the $T^{6}$ by $X_{i}^{r}, r=1, \ldots, 6$

$$
d X^{r}=2 \pi R S_{i s}^{r} d \widehat{X}^{s}+d A^{r}
$$

while the remaining one to the $S^{1}$ by $X$

$$
d X=2 \pi R m_{i r} d \widehat{X}^{r}+d A
$$

where $S_{i s}^{r} \in S p(6, Z)$ for each $\mathrm{i}=1, \ldots, 7, d A^{r}$ and $d A$ are exact one-forms. These ones are completely general without restrictions as well as the spinor fields on the target which are also scalars on the worldvolume. They carry the local degrees of freedom 
of the supermembrane. For each $T^{6}$ we provide a symplectic structure in order to define the topological condition on Section 2, they are given in Table 4.

We will denote by $\Gamma \equiv Z_{2}^{3}$ the discrete group whose elements change the sign of the maps from $\Sigma$ to $T^{7}$ according to the second row in Table 1 . We will denote by $\Lambda \equiv Z_{2}^{4}$ the discrete group whose elements change the sign of the maps from $\Sigma$ to $T^{7}$ according to the complete Table $1 . \Gamma$ is a discrete subgroup of G2 and $\Lambda$. For each sector $i$ we may associate a subgroup $Z_{2} \times Z_{2}$ of $\Gamma$ in the following way. Take on the first row of Table 1 , the triplets containing an integer $i$, for example if $i=7$ : $(1,2,7),(4,3,7),(5,6,7)$. The corresponding elements on the second row of Table 1 : $(3,4,5,6),(1,2,5,6),(1,2,3,4)$ respectively determine a subgroup $Z_{2} \times Z_{2}$ of $\Gamma$. These transformations map the sector $i$ into itself. They belong to the $S p(6, Z)$ associated to the sector. We will now show that the other elements of $\Gamma$ transform admissible maps (the ones satisfying the topological constraint) of one sector into admissible maps of another one. The integers $m_{i r}$ become determined in the procedure.

Computation of $m_{i r}$ We start with the most general expression (4.2), (4.3) in sector $i=7$, by performing a change on the homology basis and in the corresponding normalized basis of one-forms, it can always be reduced to,

$$
d \widehat{X}^{1}, d \widehat{X}^{2}, d \widehat{X}^{3}, d \widehat{X}^{4}, d \widehat{X}^{5}, d \widehat{X}^{6}, m_{7 r} d \widehat{X}^{7}
$$

where from now on we denote in a file the harmonic part of $d X^{i}$, for each $i$ ordered from 1 to 7 . To simplify the notation we do not write explicitly the $2 \pi R$ factors. The exact part is not relevant in the determination of the admissibility of a map, and may be added at any stage of the argument. If we now apply transformation $(2,4,6,7)$ the new map

$$
d \widehat{X}^{1},-d \widehat{X}^{2}, d \widehat{X}^{3},-d \widehat{X}^{4}, d \widehat{X}^{5},-d \widehat{X}^{6},-m_{7 r} d \widehat{X}^{7}
$$

is not admissible in sector 7 but it is in the other sectors. For example if we take the sector 1 , with symplectic structure given in Table 4 below, it is admissible if

$$
m_{7 r} d \widehat{X}^{r}=d \widehat{X}^{1}+m_{72} d \widehat{X}^{2}
$$

for any integer $m_{72}$.

If we now consider the transformation $(2,3,5,7)$ of $\Gamma$, (4.5) transforms into

$$
d \widehat{X}^{1},-d \widehat{X}^{2},-d \widehat{X}^{3}, d \widehat{X}^{4},-d \widehat{X}^{5}, d \widehat{X}^{6},-m_{7 r} d \widehat{X}^{7}
$$

which is admissible in sector 1 for any $m_{72}$. Under $(1,4,5,7)$ (4.5) transforms into

$$
-d \widehat{X}^{1}, d \widehat{X}^{2}, d \widehat{X}^{3},-d \widehat{X}^{4},-d \widehat{X}^{5}, d \widehat{X}^{6},-d \widehat{x}^{1}-m_{7 r} d \widehat{X}^{7}
$$


it is admissible only in sector 2 with $m_{72}=1$. Finally under $(1,3,6,7)$ (4.5) transforms into

$$
-d \widehat{X}^{1}, d \widehat{X}^{2},-d \widehat{X}^{3}, d \widehat{X}^{4}, d \widehat{X}^{5},-d \widehat{X}^{6},-d \widehat{x}^{1}-d \widehat{X}^{2} m_{7 r}
$$

which is also admissible in sector 2 . The general values of $m_{7 r}$ in order to have the full $\Gamma$ as a symmetry on the admissible set of maps is

$$
m_{7 r} d \widehat{X}^{r}=\left\{\begin{array}{l} 
\pm\left(d \widehat{X}^{1}+d \widehat{X}^{2}\right) \\
\pm\left(d \widehat{X}^{3}+d \widehat{X}^{4}\right) \\
\pm\left(d \widehat{X}^{5}+d \widehat{X}^{6}\right)
\end{array}\right.
$$

The general expression for $m_{i r}$ is obtained from $m_{7 r}$ by applying the elements of $\Gamma$.

We conclude then that given a general admissible map on any sector there always exists another admissible map which is the transformed under $\Gamma$ of the original one. The integers $m_{r}$ take some particular value in the procedure. In other words, for that particular values of $m_{r}$, the set of admissible maps is preserved under the action of $\Gamma$. Moreover, the bosonic hamiltonian as a map from the space of configurations to the reals is invariant under $\Gamma$. The same properties are valid for the discrete group $\Lambda=Z_{2}^{4}$, with the same values of $m_{r}$. However, we are considering the wrapping of the MIM2 on an oriented $T^{7}$ and the transformations on the first row of Table 1. do not preserve the orientation of $T^{7}$. We are then left only with the group of discrete symmetries $\Gamma=\left(Z_{2}^{3}\right)$. The supersymmetric hamiltonian is invariant under these symmetries. All other discrete symmetries of the hamiltonian whose bosonic part is quartic but quadratic on each map, are not symmetries of the admissible set.

The symplectic structure on each sector we have used is given in Table 4 . We notice that there is no loss of generality by using it, since on any other election of the symplectic matrices the above properties of the admissible set are also valid. The only change is on the explicit realization of the maps,

$$
\begin{aligned}
& \omega_{7}=d X^{1} \wedge d X^{2}+d X^{3} \wedge d X^{4}+d X^{5} \wedge d X^{6} \\
& \omega_{6}=d X^{2} \wedge d X^{1}+d X^{4} \wedge d X^{3}+d X^{5} \wedge d X^{7} \\
& \omega_{5}=d X^{2} \wedge d X^{1}+d X^{4} \wedge d X^{3}+d X^{7} \wedge d X^{6} \\
& \omega_{4}=d X^{2} \wedge d X^{1}+d X^{3} \wedge d X^{7}+d X^{6} \wedge d X^{5} \\
& \omega_{3}=d X^{2} \wedge d X^{1}+d X^{7} \wedge d X^{4}+d X^{6} \wedge d X^{5} \\
& \omega_{2}=d X^{1} \wedge d X^{7}+d X^{4} \wedge d X^{3}+d X^{6} \wedge d X^{5} \\
& \omega_{1}=d X^{7} \wedge d X^{2}+d X^{4} \wedge d X^{3}+d X^{6} \wedge d X^{5}
\end{aligned}
$$

Table 4: 
Remark It is important to emphasize the relation between $\Gamma$ and the $\operatorname{Sp}(6, Z)$ group of large area preserving diffeomorphisms. The space of admissible maps is invariant under the full group $S p(6, Z)$. It transforms admissible maps of one sector into admissible maps of the same sector. Its action on the harmonic sector of the maps shares in common with $\Gamma$ a subgroup $Z_{2} \times Z_{2}$ provided the maps are restricted to that sector. When the $Z_{2} \times Z_{2}$ is lifted to the whole admissible set it is not anymore a transformation generated by an area preserving diffeomorphism. However it is a symmetry, as we have shown, of the admissible set. $\Gamma$ is then the unique discrete symmetry relating the different sectors of the admissible set; the $S p(6, Z)$ acts only on each sector. We notice that these sectors arise from the different possible wrappings of the MIM2 on $T^{7}$ and their origin is not related to the twisted or untwisted sectors of the MIM2 when the identification on an orbifold is performed.

\subsection{Configuration space}

We will now define a MIM2 on the $G 2$ orbifold $T^{7} / \Gamma$ constructed by Joyce [50]. The group of transformations $\Gamma=Z_{2}^{3}$ introduced by Joyce has additional shifts with respect to the transformations in Section 3.2. Those shifts are irrelevant concerning the action of the group in the MIM2 theory since the maps only enter in terms of one-forms and hence the shifts disappear. However they are important in the construction of the orbifold. These shifts can be generated in the MIM2 theory by the constraint (2.4). It generates area preserving diffeomorphisms homotopic to the identity with infinitesimal parameters which are harmonic one-forms on $\Sigma$. The transformation for the maps is

$$
\delta X^{s}=\left\{X^{s}, \xi\right\}
$$

with $\xi=\xi_{r} \widehat{X}^{r}, \quad r=1, \ldots, 6$. The harmonic part is then shifted by (4.11)

$$
\frac{1}{\operatorname{Area}_{\Sigma}} \int_{\Sigma} \delta X^{r} \sqrt{W} d \sigma^{1} \wedge d \sigma^{2}=\omega^{r s} \xi_{s} .
$$

We may then fix six shifts, corresponding to the mean value of the map over $\Sigma$. In the notation of Section 3.2 the generators of the Joyce $Z_{2}^{3}$ are: $\alpha=(3,4,5,6), \beta=$ $(1,2,5,6), \gamma=(2,4,6,7)$ with the same shifts of value $1 / 2$. That is,

$$
\begin{array}{lr}
\alpha:\left(x^{1}, \ldots, x^{7}\right) \rightarrow & \left(x^{1}, x^{2},-x^{3},-x^{4},-x^{5},-x^{6}, x^{7}\right) \\
\beta:\left(x^{1}, \ldots, x^{7}\right) \rightarrow & \left(-x^{1},-x^{2}, x^{3}, x^{4}, 1 / 2-x^{5},-x^{6}, x^{7}\right) \\
\gamma:\left(x^{1}, \ldots, x^{7}\right) \rightarrow & \left(x^{1}, x^{2},-x^{3}, 1 / 2-x^{4},-x^{5}, 1 / 2-x^{6}, x^{7}\right) .
\end{array}
$$

The elements of the group $\Gamma$ are isometries of $T^{7}$, preserving its flat $G_{2}$-structure. The fixed points of $\alpha, \beta, \gamma$ are each 16 copies of $T^{3}$. The singular set $S$ of $\frac{T^{7}}{\Gamma}$ is a disjoint union of 12 copies of $T^{3}$. The singularity on each component of $S$ is of the 
form $T^{3} \times \frac{\mathbb{C}}{ \pm 1}$. The singularities of $T^{7} / \Gamma$ can be resolved and a metric with holonomy G2 on a compact 7 manifold may be obtained [50].

Untwisted Sector We may now consider the construction of the untwisted sector of the MIM2 on the G2 orbifold $T^{7} / \Gamma$. We start from the general space of configurations satisfying the topological constraint ensuring the irreducible wrapping of all configurations of the supermembrane. We then consider the subspace of configurations invariant under $\Gamma$. This was constructed in section 4.1. The maps are of the form (4.2) (4.3) with the restrictions on the values of $m_{i r}$ (4.10) (on that particular basis of harmonic one-forms). On the space of configurations we construct classes, two elements of a class are related by a transformation of $\Gamma$. The hamiltonian, as mentioned before has the same value on each element of the class. The untwisted sector of the theory is now defined on the space of classes. Each class represents now the map from $\Sigma$ to the orbifold. This construction may be implemented directly in the functional integral of the supermembrane, which in the case of the MIM2 has a well defined gaussian measure. The untwisted sector we have constructed breaks SUSY to $N=1$ and it is directly related to the analysis in [45].

Twisted Sector We will denote by $Z_{2}$ spin structure on a n-dimensional vector bundle $E$ a principle spin-bundle $P_{\text {Spin }}(E)$ together with a two-sheeted covering:

$$
\xi: P_{\text {Spin }}(E) \rightarrow P_{S O}(E)
$$

such that $\xi(p g)=\xi(p) \xi_{0}(g)$ for all $p \in P_{\text {Spin }}(E)$ and all $g \in$ Spin. $\xi_{0}$ is the universal covering homomorphism $\xi_{0}: \operatorname{Spin}(n) \rightarrow S O(n)$ with kernel $\{1,-1\} \sim Z_{2}$. An element of $P_{S O}(E)$ can be lifted to $P_{S p i n}(E)$ if and only if $W_{2}(p)=0$, where $W_{2}$ is the second Stiefel-Whitney class. When $n=1, P_{S O}(E)=X$ the base manifold and a spin structure is defined to be a 2 -fold covering of $X$. The $Z_{2}$ spin structures when they exist are are in one to one correspondence to a + or a - assign to the elements of a basis of homology on $X$.

We consider now the construction of the twisted sector of the M2 $2^{3}$ with central charges on a G2 manifold. The group of identifications on the target torus is $\Gamma=Z_{2}^{3}$. The twisted sectors correspond to maps which change sign when going around a cycle on $\Sigma$ according to some element of $\Gamma$. To construct all the global objects satisfying such conditions, we proceed as follows. We assign to each element of the basis of homology $\mathcal{C}_{r}, r=1, \ldots, 2 g$ an element $\Gamma_{r}$ of $Z_{2}^{3}$. Each assignment defines a $Z_{2}^{3}$ spin structure on the Riemann surface. For such spin structure we construct the following global object. The map $X^{i}, i=1, \ldots, 7$ is a section of $P_{\text {Spin }}(X)$, which is a 2 -fold covering of $X$, with a $Z_{2}$ Spin structure determined by the + or - sign assigned

\footnotetext{
${ }^{3} \mathrm{~A}$ former study of the twisted states of an extended membrane in the case of $\mathrm{M}$ theory on an orbifold $\frac{S^{1}}{Z_{2}}$ was considered in 62 .
} 
to the homology basis according to the $i^{\text {th }}$ sign \pm associated to the maps $\Gamma_{r}$. For example let us consider the $Z_{2}^{3}$ Spin-structure obtained by assigning $\Gamma_{1}=(2,3,5,7)$ to $\mathcal{C}_{1}, \Gamma_{2}=(1,4,5,7)$ to $\mathcal{C}_{2}$ and the identity $\mathbb{I}$ to the rest $\mathcal{C}_{t}, t=3,4,5,6$. We then have the corresponding transformations

\begin{tabular}{|l|l|l|l|l|l|l|l|}
\hline & $X^{1}$ & $X^{2}$ & $X^{3}$ & $X^{4}$ & $X^{5}$ & $X^{6}$ & $X^{7}$ \\
\hline $\mathcal{C}_{1} \rightarrow \Gamma_{1}$ & + & - & - & + & - & + & - \\
\hline $\mathcal{C}_{2} \rightarrow \Gamma_{2}$ & - & + & + & - & - & + & - \\
$\mathcal{C}_{3} \rightarrow \mathbb{I}$ & + & + & + & + & + & + & + \\
$\vdots$ & $\vdots$ & $\vdots$ & $\vdots$ & $\vdots$ & $\vdots$ & $\vdots$ & $\vdots$ \\
$\mathcal{C}_{6} \rightarrow \mathbb{I}$ & + & + & + & + & + & + & + \\
\hline
\end{tabular}

Table 5: The columns of the table define the sections with which the maps of the twisted sector are constructed.

We now construct the global object $X^{i}, i=1, \ldots, 7$ by considering a section of $P_{\text {Spin }}$, a 2-covering of the Riemann surface, with the $Z_{2}$ spin structure obtained by the columns in the above diagramme. The corresponding sections may be explicitly constructed in terms of the harmonic one-forms $d \widehat{X}^{1}, \ldots, d \widehat{X}^{6}$ of the $g=3$ Riemann surface:

$$
\begin{aligned}
& X^{1}=e^{\frac{i}{2} \widehat{X}^{2}} \varphi^{1}, \quad X^{2}=e^{\frac{i}{2} \widehat{X}^{1}} \varphi^{2}, \quad X^{3}=e^{\frac{i}{2} \widehat{X}^{1}} \varphi^{1}, \quad X^{4}=e^{\frac{i}{2} \widehat{X}^{2}} \varphi^{2}, \\
& X^{5}=e^{\frac{i}{2} \widehat{X}^{1}} e^{\frac{i}{2} \widehat{X}^{2}} \varphi^{5}, \quad X^{6}=\varphi^{6}, \quad X^{7}=e^{\frac{i}{2} \widehat{X}^{1}} e^{\frac{i}{2} \widehat{X}^{2}} \varphi^{7},
\end{aligned}
$$

where $\varphi^{r}, r=1, \ldots, 6$ are scalar fields wrapping $T^{7}$ as described in section 4 . These maps are scalar fields on the 2 -fold coverings of the base Riemann surface $\Sigma$. The space of those maps, for all possible assignment of elements of $Z_{2}^{3}$ to the homology basis define the twisted sector of the MIM2 theory. They remain being scalar fields, as required by the supermembrane lagrangian, but are defined on 2-fold coverings of $\Sigma$.

Remark The $S p(6, Z)$ symmetry on the admissible set is broken after identifying the points on $T^{7}$ by $\Gamma$. On each sector of the admissible set one is left with a $Z_{2} \times Z_{2}$ symmetry.

\subsection{Connection with Calabi-Yau compactifications}

It is very well known that the G2 manifold can be also built using a partial complex structure coordinate 48]. The above 3 -form can be re-expressed as

$$
\Psi=\operatorname{Re}(\Omega)+w \wedge d x_{7}
$$

In this equation $\Omega=d z_{1} \wedge d z_{2} \wedge d z_{3}$ is the complex holomorphic form of $C^{3}$ and $w=\frac{i}{2}\left(d z_{1} \wedge d \bar{z}_{1}+d z_{2} \wedge d \bar{z}_{2}+d z_{3} \wedge d \bar{z}_{3}\right)$ is the Kahler form. Since $\mathrm{SU}(3)$ is a subgroup 
of G2, one can identify the $C^{3}$ factor with a local Calabi-Yau threefold (CY3) used in two dimensional $N=2$ sigma model [51, 52, 53]. In this realization, the above three-form (4.14) is invariant under the symmetry

$$
z_{i} \rightarrow \overline{z_{i}} \quad x_{7} \rightarrow-x_{7}
$$

which is needed to ensure $N=1$ in $4 \mathrm{D}$. We will try to show that this transformation can be related to the above $Z_{2} \times Z_{2} \times Z_{2}$ symmetry used in the orbifold construction. This can be done by imposing certain constraints depending on the precise $Z_{2}$ action. Indeed, the CY3 could be taken as $T^{2} \times T^{2} \times T^{2}$ quotiented by $Z_{2} \times Z_{2}$. Since the CY condition requires the use of only two $Z_{2}$ 's $\left(Z_{2}^{1} \times Z_{2}^{2}\right)$, we need to single out the third $Z_{2}^{3}$ factor. $Z_{2}^{1} \times Z_{2}^{2}$ acts on the six-torus structure, producing as a result a CY3, and trivially on the circle $S^{1}$. The third $Z_{2}^{3}$ acts on both, the CY3 and the circle leading to the G2 structure manifold. In this way, one can identify the last action with the transformation given in (4.15).

The singularities of this orbifold can be identified with its fixed points. In the three dimensional complex factor, the fixed locus of this G2 manifold is a Lagrangian submanifold. Its volume form is defined by the real part of $\Psi$. Since the circle has two fixed points, the total singular geometry then consists of two copies of such a lagrangian submanifold. The singularities can have an interpretation in the MIM2 picture as critical points. However this does not mean that there is a degenerate locus of extremal points. On the contrary, the quantum analysis reveals that there is an absolute minimum for the hamiltonian of the supermembrane. There are no flat directions in the potential. This fact can be understood from the fact that the dual of the gauge symmetries correspond to different backgrounds and not a unique one.

Locally each singular point should be resolved like $R^{3} \times X$, where $X$ is an ALE CalabiYau 2-fold asymptotic to $C^{2} / Z_{2}$, is known as ALE space with $A_{1}$ singularity. The ALE space with $A_{1}$ singularity is described by

$$
z_{1}^{2}+z_{2}^{2}+z_{3}^{2}=0
$$

Using a simple change of variables, this is equivalent to

$$
x y=z^{2}
$$

where $x, y$ and $z$ are complex coordinates. As usual, this singularity can be removed either by deforming the complex structure or by a blow-up procedure. Geometrically, this corresponds to replacing the singular point $(x=y=z=0)$ by a $C P^{1} \sim S^{2}$. As previously explained the (APD) connected with the identity deform the shape of each $T^{2}$ and they produce translation on the orbifold side. They serve to blow up the corresponding orbifold singularities leading to a compactification on a true G2 manifold. 


\section{Quantum properties of the supersymmetric theory}

In this section we discuss the quantum consistency of our previous construction of the supersymmetric action of the supermembrane (minimally immersed) on a G2 manifold. In particular its spectrum is discrete. Both results are unique and highly nontrivial from the supermembrane point of view.

We denote the regularized hamiltonian of the supermembrane with the topological restriction by $H$, its bosonic part $H_{b}$ and its fermionic potential $V_{f}$, then

$$
H=H_{b}+V_{f}
$$

. We can define rigorously the domain of $H_{b}$ by means of Friederichs extension techniques. In this domain $H_{b}$ is self adjoint and it has a complete set of eigenfunctions with eigenvalues accumulating at infinity. The operator multiplication by $V_{f}$ is relatively bounded with respect to $H_{b}$. Consequently using Kato perturbation theory it can be shown that $H$ is self adjoint if we choose

$$
\mathrm{DomH}=\mathrm{DomH}_{b}
$$

In [40] it was shown that $\mathrm{H}$ possesses a complete set of eigenfunctions and its spectrum is discrete, with finite multiplicity and with only an accumulation point at infinity. An independent proof was obtained in [43] using the spectral theorem and theorem 2 of that paper. In section 5 of 43 a rigorous proof of the Feynman formula for the Hamiltonian of the supermembrane was obtained. In distinction, the hamiltonian of the supermembrane, without the topological restriction, although it is positive, its fermionic potential is not bounded from below and it is not a relative perturbation of the bosonic hamiltonian. The use of the Lie product theorem in order to obtain the Feynman path integral is then not justified. It is not known and completely unclear whether a Feynman path integral formula exists for this case.

In the previous sections we have provided a construction of the supermembrane with the topological restriction on an orbifold with $G_{2}$ structure that can be ultimately deformed to lead to a true G2 manifold. All the discussion of the symmetries on the Hamiltonian is performed directly in the Feynman path integral, at the quantum level, and is complete valid by virtue of our previous proofs. All other constructions in terms of supermembranes not restricted by our topological restriction are not justified in any sense and from a quantum mechanical point of view probably wrong.

In [31] action integral of the Supermembrane, the fermionic fields under the Lorentz transformations on the target space are scalars under diffeomorphisms on the worldvolume. They are scalars under area preserving diffeomorphisms, both 
connected and not connected to the identity, in the light cone gauge and there is no harmonic sector related to it. Consequently, it is invariant under all symmetries introduced in our construction and the supersymmetric theory and not only the bosonic part is compactified on the $G_{2}$ manifold.

Moreover, in [41] was proved that the theory of the supermembrane with central charges, corresponds to a nonperturbative quantization of a symplectic Super YangMills in a confined phase and the theory possesses a mass gap.

In distinction with other analysis, the discrete symmetries required to perform the orbifold with $G_{2}$ structure identification are already realized at the level of the hamiltonian leading to a top-down compactification. This fact restricts the compactification manifold to a particular one where we can guarantee that all of the above spectral properties of the supersymmetric hamiltonian compactified on a torus found before, are preserved on the compactification process on the G2 manifold, for its bosonic and supersymmetric extension, which is, a priori, a highly non-trivial fact. Indeed, the untwisted sector of the theory is exactly the same that the one corresponding to the compactification of the MIM2 on a 7-torus with the integers of the minimal immersion in the orbifold case particularized to some specific values that do not alter in any sense the spectral properties. The twisted sector of the theory only adds a finite number of states compatible with the orbifold projection, and it does neither change the spectral properties. On this $G 2$ orbifold we can guarantee the discreteness of the quantum supersymmetric spectrum of the MIM2. The deformation of the singularities is also due to the invariance of the hamiltonian under the DPA symmetries of the former theory, so there is no change in the characterization of the spectrum in the blow-up process.

\section{Phenomenological analysis of the MIM2 on this G2 mani- fold}

We will show in this section that the model we have exposed above represent a new kind of models with potential interesting properties at phenomenological level.

It has been pointed out in [11] the phenomenological interest of G2 compactifications that admit an expression in terms of CY compactifications since for those manifolds explicit metric can be obtained, i.e. [65], and ALE resolutions of the singularities may lead to interesting phenomenological properties as chirality and nonabelian gauge groups, in that sense it is very appealing to have been able to express our transformations in terms of that. It has been argued however that orbifold singularities are not enough to guarantee chirality [64], but it is needed an isolated conical singularity. Interesting models in which D6 branes are wrapping Slag cycles of a CY manifold that have an uplifting in M-theory as Taub-nut geometry with 
fractional M2 wrapping collapsed 3-cycles in a G2 compactification can be found in [66], 67, 68] with interesting phenomenological properties, also models of M2 on G2 compactifications able to produce non perturbative effects 69. Our approach at first sight could seem to not share such a nice features, however the study of its phenomenological properties is far to be closed. We would like to stress that although we have contructed a G2 manifold with orbifold singularities, we have a regular supermembrane minimally immersed on a G2 and not a fractional one. As happens in string compactifications there are different ways to obtain interesting phenomenology: let say Calabi-Yau's compactifications with Dp branes at the singularities, where the enhancement of the symmetry is due to the geometry of the singularity, that has its correspondence with the first type of models n G2 compactifications [66]-68. In those it is fundamental to have a conical singularity on the G2 compactification side. There is a second way to obtain interesting phenomenology that corresponds to have intersecting Dp branes (IIA) or magnetized Dp branes (IIB) on, for example, an orientifold orbifolded action, where the gauge and chiral properties are mainly due to the particularities of the Dp brane construction. Our M-theory model would be in correspondence with this second type. Here the chirality properties and gauge enhancement would be due to the MIM2 worldvolume properties and not associated to the former orbifolded singularities (that are smoothed). In that sense it would be interesting to compute explicitly the corresponding metric and study its phenomenological properties. Other aspects of interest like confinement from G2-manifolds 63. ( considered mainly in G2 manifolds with ALE singularities) emerge naturally in our case since the spectral properties of the MIM2 have not changed when we have performed the identification in the target space and the theory shows confinement. In [41], it is argued how the MIM2 theory could reproduce the strong coupling regime of susy QCD since there are present glueballs and it possesses a discrete spectrum with a mass gap. Indeed it corresponds exactly to a symplectic Super Yang-Mills in 4d coupled to several scalar fields. The proposal is that the confined phase of the theory corresponds to the MIM2 on a 7-torus and the quark-gluon plasma phase to the ordinary M2 compactified in a 7-torus. Both phases are connected through a topological phase transition of quantum origin that breaks the center of the group. Since the theory of MIM2 on a G2 manifold do not change its quantum spectral properties, those previous properties would apply and it could also described the confined phase of the theory. Regarding moduli stabilization aspects, assuming the target torus is fixed to be isotropic, the moduli parametrizing the position of the MIM2 on a 7 -torus as well as the overall moduli parametrizing the size of the manifold is fixed [45]. When the MIM2 is compactified on the G2 orbifold the singularities are resolved through a backreaction effect due to the wrapping, then the moduli associated to those singularities we argue that they are also fixed. We have then obtained the 11D supermembrane minimally immersed on a particular G2 manifold. 


\section{Discussion and conclusions}

In this paper we have shown, for first time up to our knowledge, a top-down compactification of the supermembrane on a particular G2 manifold. The 11D supermembrane theory restricted by a topological condition due to an irreducible wrapping is stable at classical and quantum level, has discrete spectrum and a mass gap. It can be compactified on a $\frac{T^{7}}{Z_{2}^{3}}$ orbifold preserving its quantum stability properties. The resulting theory can be interpreted as a compactification on a G2 manifold. Indeed, the symmetries of the theory produce a holonomy bundle that corresponds exactly to those associated to the Riemanian holonomy of a G2 manifold. By performing the identification on the target space of the discrete symmetries preserving the topological condition, only those symmetries associated to the G2 orbifold space are possible, neither the configuration states nor the minimal immersions are invariant under the spureous symmetries that would break the supersymmetry to $N=0$. One can see that the holonomy bundle associated to the compactification to $5 \mathrm{D}$ is related with the Klein subgroup. When this is further compactified to the remaining $S^{1}$ there exist seven possible immersions of the M2-brane on the target space of the $T^{7}$ that allow to make exactly the identifications with the G2 holonomy group. The singularities of this G2 orbifold may be resolved, as shown by Joyce, leading to a true G2 manifold. The shifts have their origin in the diffeomorphisms homotopic to the identity of the MIM2. The untwisted and twisted sector are completely characterized. Moreover, this result can also be seen in terms of a $\frac{C Y_{3} \times S^{1}}{Z_{2}}$.

We can finally conclude that for first time a consistent quantization procedure for the supermembrane on a G2 manifold has been presented. It is in terms of the supersymmetric action of the supermembrane, subject to a topological condition -which is equivalent to have central charges due to an irreducible winding-, on a particular G2 manifold.

From a phenomenological point of view, the supermembrane with central charges on the $G_{2}$ manifold represents a new kind of models of compactification in which the supermembrane is minimally immersed a long the whole G2 manifold and not just at the singularities. Typically in the literature there has been studied the wrapping of M2s around the singularities of a $G_{2}$ manifold, in analogy with the constructions of Dp-branes models at singular Calabi-Yaus in string theories. These constructions require particular conditions in order to obtain interesting properties, i.e. chirality is associated to the existence of conical singularities on the $G_{2}$ manifold and the gauge groups to have orbifold singularities: ADE singularities, etc. In the supermembrane with central charges the gauge field content is already defined on its worldvolume and is not associated to the singularities of the compactification manifold. Since there is also a flux condition on the worldvolume, chirality in our model cannot be 
automatically ruled out -in resemblance with the magnetized D-brane models on type II constructions- and deserve further study. We think that the supermembrane with central charges compactified on this $G_{2}$ manifold is then an interesting starting point on the construction of a new kind of models with potentially rich phenomenology.

\section{Acknowledgements}

We thank L. J. Boya, G. Bonelli, C. Hull, A. Lerda, H. Nicolai, M. Petropoulos, N. Prezas, J. Roseel, V. N. Suryanarayana, I. Stavrov, A. Uranga, for helpful discussions. MPGM gives thanks to Departamento de Fisica, Universidad de Zaragoza, Spain, for kind invitation and hospitality while part of this work was done. AB would like to thank UFR-Lab/ PHE, Rabat for hospitality where a part of this work was done, and the project, between Universidad de Zaragoza (Spain) and Faculte des Sciences de Rabat (Morocco), Fisica de altas energias: Particulas, Cuerdas y Cosmologia (grant A9335/07). MPGM is partially supported by Dipartimento di Fisica di Universita di Torino under European Comunity's Human Potential Programme and by the Italian MUR under contracts PRIN-2005023102 and PRIN-2005024045. The work of AR is partially supported by a grant from MPG, Albert Einstein Institute, Germany and by PROSUL, under contract CNPq 490134/2006-08. The work of AB and AS has been supported by CICYT (grant FPA-2006-02315) and DGIID-DGA (grant 2007-E24/2), Spain.

\section{References}

[1] P. K. Townsend and G. Papadopoulos, Compactification of D=11 supergravity on spaces of exceptional holonomy, Phys. Lett B 357 (1995)472, hep-th/9506150.

[2] B. Acharya, On realizing $N=1$ super Yang-Mills in M-theory, hep-th/0011089.

[3] J. A. Harvey, G. Moore, Superpotentials and Membrane Instantons, hep-th/9907026.

[4] S. Kachru, J. McGreevy, M-theory on Manifolds of G2 Holonomy and Type IIA Orientifolds, JHEP 0106 (2001) 027, hep-th/0103223.

[5] M. F. Atiyah and E. Witten, M-theory dynamics on a manifold of G2 Holonomy, hep-th/0107177.

[6] L. B. Anderson, A. B. Barrett, A. Lukas, M. Yamaguchi, Four-dimensional Effective M-theory on a Singular G(2) Manifold, Phys. Rev.D74:086008, 2006, hep-th/0606285.

[7] E. Witten, Anomaly Cancellation On Manifolds Of G2 Holonomy, hep-th/0108165.

[8] B. Acharya, E. Witten, Chiral Fermions from Manifolds of G2 Holonomy, hep-th/0109152. 
[9] A. Belhaj, L.B. Drissi, J. Rasmussen, On N=1 gauge models from geometric engineering in M-theory, Class. Quant. Grav.20 (2003) 4973-4982, hep-th/0304019.

[10] A. Belhaj, On manifolds of G(2) holonomy in M-theory compactifications, Afr.J. Math. Phys. 1(2004) 61-68.

[11] B. S. Acharya, S. Gukov $M$ theory and Singularities of Exceptional Holonomy Manifolds Phys.Rept. 392 (2004) 121-189, hep-th/0409191.

[12] M. Cicoli, J. P. Conlon, F. Quevedo, Systematics of String Loop Corrections in Type IIB Calabi-Yau Flux Compactifications, arXiv:0708.1873 [hep-th].

[13] V. Balasubramanian, P. Berglund, J. P. Conlon, F. Quevedo, Systematics of Moduli Stabilisation in Calabi-Yau Flux Compactifications, JHEP 0503 (2005) 007, hep-th/0502058.

[14] F. Denef, M. R. Douglas, S. Kachru, Physics of String Flux Compactifications, arXiv:hep-th/0701050.

[15] J.F.G. Cascales, M.P. Garcia del Moral, F. Quevedo, A. Uranga, Realistic D-Brane Models on Warped Throats: Fluxes, Hierarchies and Moduli Stabilization, JHEP 0402 (2004) 031, hep-th/0312051.

[16] J.F.G. Cascales, A.M. Uranga, Chiral 4d string vacua with D-branes and NSNS and $R R$ fluxes, JHEP 0305 (2003) 011 hep-th/0303024.

[17] G. Curio, A. Klemm, D. Lust, S. Theisen, On the vacuum structure of type II string compactifications on Calabi-Yau spaces with H fluxes. Nucl.Phys.B 609 (2001), 3-45, hep-th/0012213.

[18] R. Blumenhagen, B. Kors, D. Lust, S. Stieberger, Four-dimensional String Compactifications with D-Branes, Orientifolds and Fluxes, hep-th/0610327.

[19] C. M. Hull, R. A. Reid-Edwards, Flux Compactifications of M-Theory on Twisted Tori, JHEP 0610 (2006) 086, arXiv:hep-th/0603094.

[20] G. Dall'Agata, R. D'Auria, S. Ferrara, Compactifications on twisted tori with fluxes and free differential algebras, Phys. Lett. B619 (2005) 149-154, hep-th/0503122.

[21] G. Dall'Agata, S. Ferrara, Gauged supergravity algebras from twisted tori compactifications with fluxes, Nucl. Phys. B717 (2005) 223-245, arXiv:hep-th/0502066.

[22] B. Acharya, K. Bobkov, G. Kane, P. Kumar, D. Vaman, An M theory Solution to the Hierarchy Problem, Phys.Rev.Lett. 97 (2006) 191601, hep-th/0606262.

[23] B.S. Acharya, K. Bobkov, G.L. Kane, P. Kumar, J. Shao The $G_{2}-M S S M-A n M$ Theory motivated model of Particle Physics arXiv:0801.0478 [hep-th]. 
[24] J.P. Conlon, C.H. Kom, K. Suruliz, B.C. Allanach, F. Quevedo, Sparticle Spectra and LHC Signatures for Large Volume String Compactifications. JHEP 0708:061,2007.arXiv:0704.3403 [hep-ph].

[25] C.M. Hull, Doubled Geometry and T-Folds, JHEP 0707 (2007)080, hep-th/0605149.

[26] A. Dabholkar, C. Hull, Duality twists, orbifolds, and fluxes, JHEP 0309 (2003) 054, hep-th/0210209.

[27] A. Flournoy, B. Wecht, B. Williams, Constructing nongeometric vacua in string theory, Nucl. Phys. B 706 (2005) 127-149, hep-th/0404217.

[28] G. Dall'Agata, N. Prezas, Scherk-Schwarz reduction of M-theory on G2-manifolds with fluxes, JHEP 0510 (2005) 103, hep-th/0509052.

[29] R. Blumenhagen, L. Gorlich, B. Kors, D. Lust, Asymmetric orbifolds, noncommutative geometry and type I string vacua, Nucl. Phys. B 582(2000) 44-64, hep-th/0003024.

[30] C.M. Hull, A Geometry for non-geometric string backgrounds JHEP 0510 (2005) 065, hep-th/0406102.

[31] E. Bergshoeff, E. Sezgin, P.K. Townsend, Supermembranes and eleven-dimensional supergravity, Phys. Lett. B189 (1987) 75-78.

[32] B. de Wit, J. Hoppe, H. Nicolai, On the quantum mechanics of supermembranes, Nucl. Phys. B305 (1988) 545.

[33] B. de Wit, M. Luscher, H. Nicolai, The supermembrane is unstable, Nucl. Phys. B320 (1989) 135.

[34] B. de Wit, U. Marquard, H. Nicolai, Area preserving diffeomorphisms and supermembrane lorentz invariance, Commun. Math. Phys. 128 (1990) 39-62.

[35] B. de Wit, K. Peeters, J. Plefka, Supermembranes with winding, Phys. Lett. B409 (1997) 117-123, hep-th/9705225.

[36] I. Martin, A. Restuccia, R. S. Torrealba, On the stability of compactified $D=11$ supermembranes, Nucl. Phys. B521 (1998) 117-128, hep-th/9706090.

[37] I. Martin, J. Ovalle, A. Restuccia, D-branes, symplectomorphisms and noncommutative gauge theories, Nucl. Phys. Proc. Suppl. 102(2001) 169-175. Compactified $D=11$ supermembranes and symplectic noncommutative gauge theories, Phys. Rev. D64 (2001) 046001, hep-th/0101236.

[38] M.P. Garcia del Moral, A. Restuccia, On the spectrum of a noncommutative formulation of the $D=11$ supermembrane with winding, Phys. Rev. D66 (2002) 045023, hep-th/0103261. 
[39] L. Boulton, M. P. Garcia del Moral, I. Martin, A. Restuccia On the spectrum of a matrix model for the $D=11$ supermembrane compactified on a torus with non-trivial winding, Class. Quant. Grav. 19 (2002) 2951, hep-th/0109153.

[40] L. Boulton, M.P. Garcia del Moral, A. Restuccia, Discreteness of the spectrum of the compactified $D=11$ supermembrane with non-trivial winding, Nucl. Phys. B671 (2003) 343-358, hep-th/0211047.

[41] L. Boulton, M.P. Garcia del Moral, A. Restuccia, The Supermembrane with central charges: (2+1)-D NCSYM, confinement and phase transition, hep-th/0609054.

[42] J. Bellorin, A. Restuccia, D=11 Supermembrane wrapped on calibrated submanifolds, Nucl. Phys. B737 190-208, 2006, hep-th/0510259.

[43] L. Boulton and A. Restuccia, The Heat kernel of the compactified D=11 supermembrane with non-trivial winding, Nucl. Phys. B724 (2005) 380-396, hep-th/0405216.

[44] B. de Wit, K. Peeters, J. Plefka, Superspace Geometry for Supermembrane Backgrounds, Nucl. Phys. B532 (1998) 99-123, arXiv:hep-th/9803209.

[45] M.P. Garcia del Moral, J.M. Pena, A. Restuccia, N=1 $4 D$ Supermembrane from 11D, arXiv:0709.4632 [hep-th].

[46] M.P. Garcia del Moral, I. Martin, A. Restuccia, Nonperturbative SL(2,Z) $(p, q)$-strings manifestly realized on the quantum M2. arXiv:0802.0573 [hep-th]

[47] A. Belhaj, M. P. Garcia del Moral, On Non Commutative G2 structure, J. Phys. A38 (2005) 2773-2784, hep-th/0408011.

[48] R. Roiban, C. Romelsberger, J. Walcher, Discret Torsion in Singular $G_{2}$-Manifolds and Real LG, Adv. Theor. Math. Phys. 6 (2003) 207-278, hep-th/0203272.

[49] O.P. Santillan, A Construction of G(2) holonomy spaces with torus symmetry, Nucl.Phys. B660 (2003)169-193, hep-th/0208190.

[50] D. Joyce, Compact manifolds of Special Holonomy, Oxford University Press, 2000.

[51] E. Witten, Phases of $N=2$ theories in two dimensions, Nucl. Phys. B 403 (1993) 159, hep-th/9301042.

[52] M. Aganagic, C. Vafa, Mirror symmetry, D-branes and counting holomorphic discs, hep-th/0012041.

[53] A. Belhaj, Mirror symmetry and Landau Ginzburg Calabi-Yau superpotentials in F-theory compactifications, J. Phys. A 35 (2002) 965, hep-th/0112005.

[54] J. Giraud, Cohomologie non-abelienne, volume 179 of Grundl. Springer Verlag, Berlin,1997. 
[55] J.L. Brylinski, Prog. in Math, Vol, 107, Loop Spaces Characteristic Clases and Geometric Quantization, Birkhäuser, Boston (1993).

[56] M. Caicedo, I. Martín, A. Restuccia, Gerbes and duality, Annals Phys. 300 (2002), 32-53, arXiv:hep-th/0205002.

[57] J. C. Baez, U. Schreiber, Higher gauge theory, math/0511710.

[58] J. C. Baez, Higher Yang-Mills theory, hep-th/0206130.

[59] J.M. Isidro, Gerbes, quantum mechanics and gravity, hep-th/0510075.

[60] M. Mackaay, R. Picken, The Holonomy of gerbes with connections, math/0007053.

[61] T. Zehra Husain, M2-branes wrapped on holomorphic curves, JHEP 0312 (2003) 037, hep-th/0211030.

[62] P. Horava, E. Witten, Heterotic and type I string dynamics from eleven-dimensions, Nucl.Phys.B460 (1996)506-524, hep-th/9510209.

[63] B.S. Acharya, Confining Strings from $G_{2}$-holonomy spacetimes arXiv:hep-th/0101206.

[64] B. Acharya, E. Witten, Chiral fermions from manifolds of G(2) holonomy arXiv: hep-th/0109152.

[65] J.D. Edelstein, A. Paredes, A.V. Ramallo, Let's twist again: General metrics of G(2) holonomy from gauged supergravity JHEP 0301 (2003) 011, arXiv: hep-th/0211203.

[66] J. D. Edelstein, C. Nunez, D6-branes and $M$ theory geometrical transitions from gauged supergravity JHEP 0104 (2001) 028, arXiv: hep-th/0103167.

[67] M. Atiyah, J. M. Maldacena, C. Vafa, An M theory flop as a large $N$ duality J.Math.Phys.42 (2001) 3209-3220, arXiv: hep-th/0011256.

[68] M. Cvetic, G. Shiu, A. M. Uranga, Chiral type II orientifold constructions as $M$ theory on G(2) holonomy spaces Dubna 2001, Supersymmetry and unification of fundamental interactions* 317-326, arXiv: hep-th/0111179.

[69] J. A. Harvey, G. W. Moore, Superpotentials and membrane instantons arXiv: hep-th/9907026. 